\title{
On Jupiter's inertial mode oscillations
}

\author{
B. Dintrans ${ }^{1,2, \star}$ and R. Ouyed ${ }^{1}$ \\ 1 Nordic Institute for Theoretical Physics, Blegdamsvej 17, 2100 Copenhagen, Denmark \\ 2 Laboratoire d'Astrophysique de Toulouse, Observatoire Midi-Pyrénées, 14 avenue É. Belin, 31400 Toulouse, \\ France
}

Received 21 June 2001 /Accepted 8 July 2001

\begin{abstract}
Properties of inertial modes of Jupiter are investigated for an $n=1$ polytropic description of the planet interior. We use the anelastic approximation to overcome the usual handicap of a severe spherical harmonics truncation. A powerful iterative solver then allows us to compute the frequencies of the most promising low-order modes using as many spherical harmonics as necessary. The induced $\mathcal{O} 1 \%$ errors of our model are now within observational limits. A plausible seismological model of Jupiter might thus be at hand provided the use of a more realistic description of the planet interior.
\end{abstract}

Key words. planets and satellites: individual: Jupiter

\section{Introduction}

Detecting global Jovian oscillations is still a major challenge since, even with the most recent detection techniques, no acoustic or gravity modes have been convincingly identified (Schmider et al. 1991; Mosser et al. 1993, 2000; Cacciani et al. 2001). Furthermore, the collision of the Shoemaker-Levy9 fragments was unfortunately not energetic enough as to allow any positive detection of primary waves (Walter et al. 1996; Mosser et al. 1996).

Upcoming observational projects, such as JOVIS (Baglin \& Mosser 1999), open a new window on the planet dynamics with the possibility of detecting long-period mode oscillations. Because of Jupiter's rapid rotation, inertial modes would be good candidates. These have first been investigated by Lee \& Saio (1990) and Lee et al. (1992) (hereafter LSVH) using different models for the planet. Their approach was nonetheless limited by the use of no more than three spherical harmonics in the angular description of the solutions (see Sect. 2.1). We have today the numerical tools to tackle the same problem by including as many terms as necessary in each of the spherical harmonic expansions (Rieutord \& Valdettaro 1997; Dintrans et al. 1999).

The plan of the paper is as follows: in Sect. 2 we introduce our model, its specificity and the equations we solve for. In Sect. 3, we discuss mode identification methods in presence of rotation and show how the inclusion of

Send offprint requests to: B. Dintrans,

e-mail: dintrans@nordita.dk

* Supported by the European Commission under MarieCurie grant No. HPMF-CT-1999-00411. diffusion allows us to discriminate the inertial eigenmodes in terms of least/high damped ones. Our results are then presented in Sect. 4 before concluding in Sect. 5 .

\section{Our model}

\subsection{Specificity of our study}

The properties of inertial mode oscillations are commonly investigated by first expanding each perturbation in a serie of spherical harmonics. However, because of the Coriolis couplings between the harmonics $(\ell, \ell \pm 1)$, one is faced with an infinite system of coupled ordinary differential equations. Such a system is challenging in its coding and requires prohibitive memory. LSVH solved these two aspects by considering at most the first three terms in each of the spherical harmonic expansions. They nevertheless recognized the necessity of calculations with larger numbers of spherical harmonics before detailed comparisons could be made between the theoretically calculated frequencies and the yet to be measured oscillations of Jupiter. Indeed, for the higher overtones, truncation errors as high as 30\% were reached when two harmonics instead of three were used.

In our case, the memory requirement is immediately dealt with by adopting the anelastic approximation (see also Dintrans \& Rieutord 2000). The idea is to filter out the acoustic waves in the infinite system while keeping the density variations across the planet. This is further justifiable since such high-frequency waves are irrelevant to the dynamics of the inertial modes. Remains the coding aspect of the problem which is taken care of by a preprocessing 
Perl program capable of managing as many spherical harmonics as necessary. Errors in our approach are thus mainly induced by the use of the anelastic approximation and are estimated to be $\mathcal{O} 1 \%$ (see Dintrans \& Rieutord 2001).

\subsection{The oscillation equations under the anelastic approximation}

Assuming the time-dependence of the eigenmodes to be $\exp i \sigma t$ (where $\sigma$ is the angular frequency in units of $\operatorname{rad~} \mathrm{s}^{-1}$ ), the anelastic equations for the linear perturbations in the co-rotating frame are given by

$\left\{\begin{array}{l}i \sigma \boldsymbol{u}+2 \boldsymbol{\Omega} \times \boldsymbol{u}=-\boldsymbol{\nabla}\left(P^{\prime} / \rho_{0}\right), \\ \operatorname{div}\left(\rho_{0} \boldsymbol{u}\right)=0,\end{array}\right.$

where $\boldsymbol{u}$ is the velocity, $P^{\prime}$ the Eulerian pressure perturbation, $\Omega$ the angular frequency $(2 \boldsymbol{\Omega} \times \boldsymbol{u}$ being the Coriolis force) and $\rho_{0}$ denotes the equilibrium density of our interior model of Jupiter which we describe by the $n=1$ polytrope (Hubbard 1984). As boundary conditions, we impose the regularity of the velocity at the centre while the anelastic approximation implies that the radial component of the velocity vanishes at the surface (see Dintrans \& Rieutord 2001).

Using the spherical coordinate system $(r, \theta, \varphi)$, we expand the velocity on spherical harmonics $Y_{\ell}^{m}(\theta, \varphi)$ (Rieutord 1991). One arrives at an infinite set of coupled radial equations to be solved as a generalized eigenvalue problem of the form

$\mathcal{M}_{\mathrm{A}} \boldsymbol{\psi}_{m^{ \pm}}=\lambda \mathcal{M}_{\mathrm{B}} \boldsymbol{\psi}_{m^{ \pm}}$

where $\lambda=i \sigma$ is the complex eigenvalue associated with eigenvector $\boldsymbol{\psi}_{m^{ \pm}}$. The $m^{+}$-modes corresponds to even solutions $(\ell=|m|,|m|+2,|m|+4, \ldots)$ whereas $m^{-}$modes are odd ones $(\ell=|m|+1,|m|+3,|m|+5, \ldots)$. We note that even (odd) modes define solutions that are symmetric (antisymmetric) with respect to the equatorial plane. Finally, this eigenvalue problem is discretized on the Gauss-Lobatto grid associated with Chebyshev polynomials yielding to matrices $\mathcal{M}_{\mathrm{A}}$ and $\mathcal{M}_{\mathrm{B}}$ of order about $L \times(N+1)$ when $L$ spherical harmonics and $N$ polynomials are considered.

\section{Mode identification in presence of rotation}

In this section we discuss the notion of mode identification when adding rotation to the $p$ - and $g$-modes as compared to the case of inertial modes.

\section{1. $p$ - and g-modes}

One recalls that two cases need to be considered depending on the non-rotating frequency shift $\sigma_{k \ell}(k$ being the radial order) with respect to the Coriolis frequency $2 \Omega$ :

i) $\sigma_{k \ell} \gg 2 \Omega(p$ - and low-order $g$-modes): the weak Coriolis couplings can be described by a perturbative theory (i.e. $\left.\sigma_{k \ell m} \simeq \sigma_{k \ell}+m C_{k \ell} \Omega\right)$ making the $(k, \ell, m)$ identification possible;

ii) $\sigma_{k \ell} \lesssim 2 \Omega$ (high-order $g$-modes): the strong Coriolis couplings prevent any $\ell$-identification of modes. A pulsation mode cannot henceforth be identified by a set $(\ell, m)$ since the description of its angular dependence in terms of a single spherical harmonic $Y_{\ell}^{m}(\theta, \varphi)$ is not possible (Dintrans et al. 1999).

\subsection{Inertial modes}

Only the $\sigma_{k \ell} \leq 2 \Omega$ regime exists, i.e. the spectrum is bounded by the Coriolis frequency. The Coriolis couplings are dominant over the entire spectrum making the $\ell$ identification meaningless. Although they recognized this fact, LSVH chose "for simplicity" to attribute a given $\ell$-value to their inertial eigenmodes; i.e. they arranged their low-order solutions in terms of $\ell=1,2,3,4$ for $m=-1,-2$, and $m=-3$ with eigenvalues sorted in decreasing values. This arrangement is in fact unappropriate, as shown for example with the eigenmodes of the incompressible rotating sphere (Bryan 1889): for $m=0^{+}$, the first four low-order frequencies are $\sigma / 2 \Omega=$ $[0.654 ; 0.830 ; 0.469 ; 0.677]$ with no possible $\ell$-attribution (see also Greenspan 1969).

\subsection{Identification from the $(|\tau|, \sigma)$-diagram}

Low-degree and low-order $p$ - or $g$-modes are commonly identified in the computed spectrum by plotting the $(\ell, \sigma)$ diagram. Unfortunately, such a tool cannot be used for inertial modes since $\ell$ is not a suitable quantum number anymore.

However, one would expect that the most promising observational inertial modes are the ones with the weakest Coriolis couplings. Such modes, hereafter referred to as "low-order" modes, only involve a small family of spherical harmonics and are for this reason associated to the smallest resolutions $L$. Therefore the challenge is to extract them among the entire computed modes defining the spectrum, thus the question: "at a given $m$ and frequency shift $\sigma$, what is the inertial mode which involves the least of spherical harmonics, that is, the one whom the $\ell$-couplings are the weakest?". As we will prove in Sect. 4.2 and in more details in a forthcoming paper, one way to extract the modes in question consists in plotting a $(|\tau|, \sigma)$-diagram, $\tau$ being the (negative) damping rate of the mode when adding a slight diffusion.

\section{Results}

Hereafter dimensionless angular eigenfrequencies $\widetilde{\sigma}$ and damping rates $\widetilde{\tau}$ are given in units of $2 \Omega$, that is $\sigma=2 \Omega \widetilde{\sigma}$ and $\tau=2 \Omega \widetilde{\tau}$. For Jupiter, it means that oscillation frequencies are $\nu(\mathrm{mHz})=\sigma / 2 \pi \simeq 0.056 \widetilde{\sigma}$ whereas periods are $T(\mathrm{~h})=2 \pi / \sigma \simeq 4.958 / \widetilde{\sigma}$. 


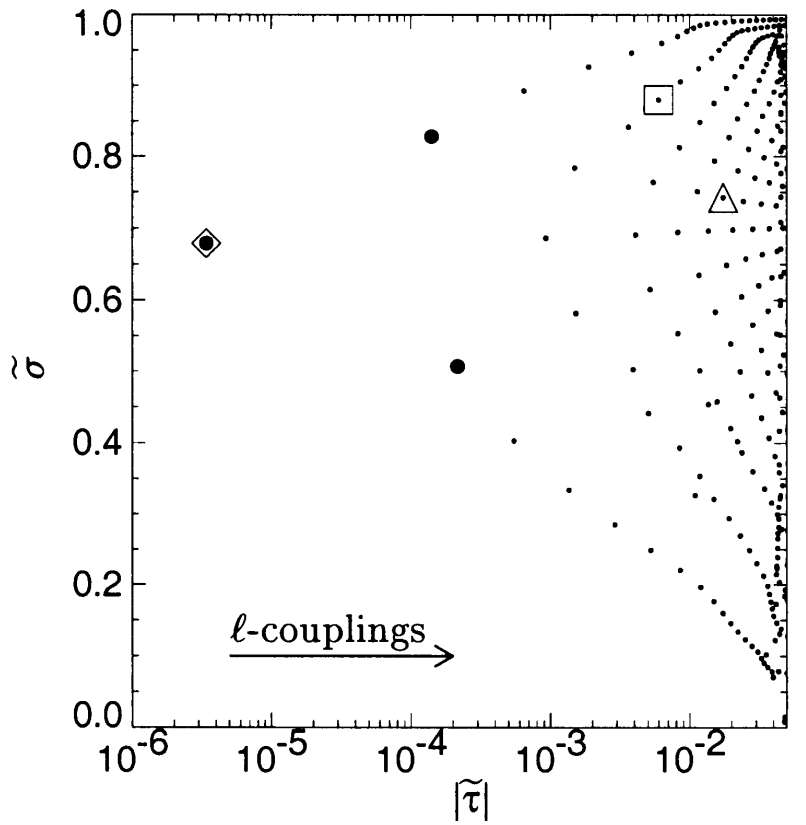

Fig. 1. The $(|\widetilde{\tau}|, \widetilde{\sigma})$-diagram for the $m=0^{+}$eigenvalues, where $\tilde{\tau}$ and $\tilde{\sigma}$ are the dimensionless damping rate and frequency, respectively. The thick dots to the left denote the first three low-order modes. The diamond, square and triangle modes, chosen in order of increasing $\ell$-couplings (arrow), are used when discussing truncation effects in Sect. 4.2.

\subsection{Low-order modes for $m=-1^{+}, 0^{+}, 1^{+}$}

We add a small viscosity term to the momentum equation in order to organize the eigenvalues in terms of least/high damped pulsation modes. By plotting them in the $(|\widetilde{\tau}|, \widetilde{\sigma})$ diagram, we are able to identify the low-order modes which position themselves in the left region of the plot. An example is shown in Fig. 1 for the $m=0^{+}$eigenvalues where few low-order modes are depicted by thick dots. This figure was obtained using the QZ algorithm with the resolution $L=N=60$ and an Ekman number $E=10^{-5}$ ( $E=\nu / 2 \Omega R^{2}$, where $\nu$ is the kinematic viscosity and $R$ the Jupiter radius).

Once the best low-order modes isolated, remains the important task/step of accurate computation of the corresponding eigenfrequencies in the astrophysical relevant limit of zero viscosity. The QZ algorithm is not the most suitable for this job because the matrix band structure of $\mathcal{M}_{\mathrm{A}}$ and $\mathcal{M}_{\mathrm{B}}$ is not taken advantage of; i.e. Fig. 1

Table 1. Dimensionless frequencies $\tilde{\sigma}$ of the first five low-order modes for $m=-1^{+}, 0^{+}, 1^{+}$. The associated oscillation periods (in hours) are given in parenthesis.

\begin{tabular}{ccc}
\hline$-1^{+}$ & $0^{+}$ & $1^{+}$ \\
\hline $0.656(7.558)$ & $0.679(7.302)$ & $0.706(7.023)$ \\
$0.201(24.67)$ & $0.828(5.988)$ & $0.863(5.745)$ \\
$0.799(6.205)$ & $0.507(9.779)$ & $0.719(6.896)$ \\
$0.491(10.10)$ & $0.403(12.30)$ & $0.921(5.383)$ \\
$0.327(15.16)$ & $0.893(5.552)$ & $0.343(14.45)$ \\
\hline
\end{tabular}

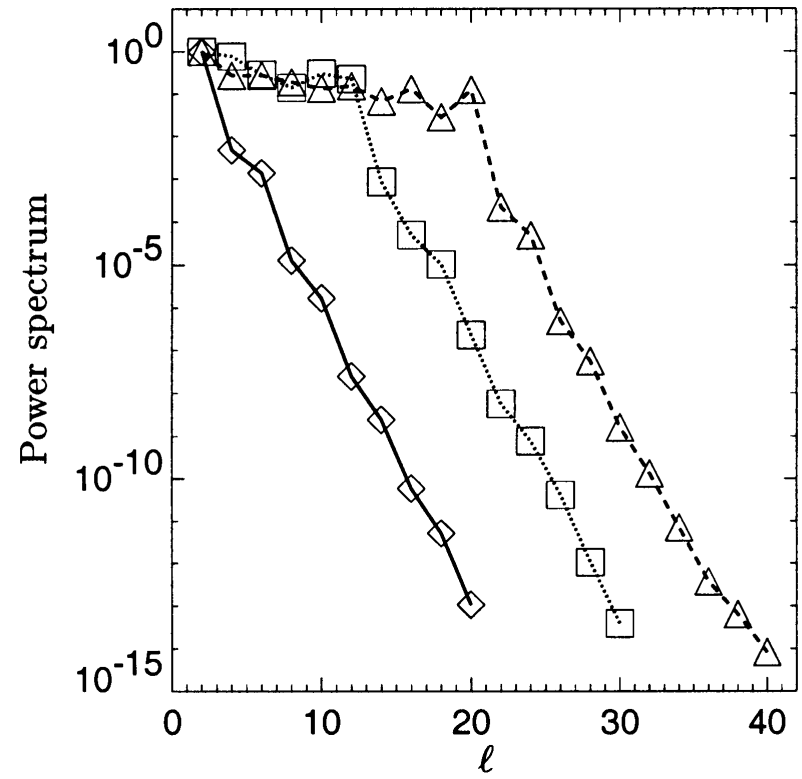

Fig. 2. Normalized angular power spectra of the radial component $u_{\mathrm{r}}$ of the velocity for the three modes labelled by a diamond, a square and a triangle in Fig. 1. Note the increase of the $\ell$-couplings with the mode order.

required 1.8 Gbytes of memory and 16 hours of computation on Origin 2000.

We use an Arnoldi-Chebyshev iterative solver to extract the interesting eigenvalues lost in the dense spectrum at $E=0$. Taking advantage of the matrix band structure, it leads to substantial gain of both memory and computing time. Table 1 shows the computed frequencies of the first five low-order modes for the $m=0^{+}$ and $m= \pm 1^{+}$cases. As can be noted from the associated periods given in parenthesis (in hours), low-order inertial modes - which must always exceed $T_{\text {rot }} / 2 \sim 4.958 \mathrm{~h}$ - can be as high as one day. It is thus clear that these very long period modes require continuous observation using either a ground-based network of dedicated telescopes or, yet better, a devoted satellite such as the mentioned JOVIS project.

\subsection{Truncation effects}

In order to test the effect of the harmonic truncation, we compute, in the limit $E=0$, the $\ell$-spectra of the three modes denoted by a diamond, a square and a triangle in Fig. 1. That is, for each $\ell$, the maximum value over the Chebyshev coefficients of $u_{\mathrm{r}}$ is plotted.

Figure 2 shows that only few spherical harmonics are necessary to describe the lowest-order eigenmode (diamond) whereas many more harmonics are required to resolve the square and triangle ones. To reach a relative error of $\mathcal{O}\left(10^{-5}\right)$, we indeed checked that the diamond mode only requires $L=4$ spherical harmonics while the square and triangle modes necessitate at least $L=14$ and $L=22$ harmonics, respectively. That is, eigenvalues are 
extremely sensitive to $L$-values before the sharp drop in the curves of Fig. 2.

Combining our findings from Figs. 1 and 2, the link between the damping rate of an inertial mode and the strength of its $\ell$-couplings is clearly demonstrated: the higher the damping rates, the higher the $\ell$-couplings, thus the higher the needed resolution $L$. It well justifies the use of the $(|\widetilde{\tau}|, \widetilde{\sigma})$-diagram to identify the low-order inertial modes and to remedy to the fact that $\ell$ is no longer a suitable quantum number.

\section{Conclusion}

We investigated the properties of the inertial modes of Jupiter for an $n=1$ polytropic model of the planet. Our calculations have been performed with as many spherical harmonics as necessary to resolve eigenmodes in the anelastic approximation.

We showed that adding a slight viscosity to the equations allows us to identify what we referred to as "loworder" modes, that is inertial modes which only involve a few spherical harmonics and are the most promising for observations. Once these low-order modes identified, we compute them in the relevant adiabatic limit of zero viscosity. We also showed that, while the lowest-order mode is fully described by four spherical harmonics, the following low-order modes require many more harmonics (up to twenty) as a consequence of the succeeding Coriolis couplings.

An avenue for future work consists on taking into account density discontinuities in the planet's interior (such as the so-called PPT or the plasma phase transition; Stevenson \& Salpeter 1976; Zharkov \& Trubitsyn 1976) and the radiative window at the surface (Guillot et al. 1994).
Acknowledgements. We thank M. Rieutord and B. Mosser for helpful comments and L. Valdettaro and M. Rieutord for letting us use their package Linear Solver Builder.

\section{References}

Baglin, A., \& Mosser, B. 1999, BAAS, 31 (DPS meeting 31, 08.13)

Bryan, G. 1889, Phil. Trans. R. Soc. London, 180, 187

Cacciani, A., Dolci, M., Moretti, P. F., et al. 2001, A\&A, 372, 317

Dintrans, B., Rieutord, M., \& Valdettaro, L. 1999, J. Fluid Mech., 398, 271

Dintrans, B., \& Rieutord, M. 2000, A\&A, 354, 86

Dintrans, B., \& Rieutord, M. 2001, MNRAS, 324, 635

Guillot, T., Chabrier, G., Morel, P., \& Gautier, D. 1994, Icarus, 112,354

Greenspan, H. P. 1969, The theory of rotating fluids (Cambridge University Press)

Hubbard, W. B. 1984, Planetary Interiors (Van Nostrand Reinhold Co., New York)

Lee, U., \& Saio, H. 1990, ApJ, 359, L29

Lee, U., Strohmayer, T. E., \& van Horn, H. M. 1992, ApJ, 397, $674(\mathrm{LSVH})$

Mosser, B., Mékarnia, D., Maillard, J. P., et al. 1993, A\&A, 267,604

Mosser, B., Galdemard, P., Lagage, P., et al. 1996, Icarus, 121, 331

Mosser, B., Maillard, J. P., \& Mékarnia, D. 2000, Icarus, 144, 104

Rieutord, M. 1991, Geophys. Astrophys. Fluid Dyn., 59, 185

Rieutord, M., \& Valdettaro, L. 1997, J. Fluid Mech., 341, 77

Schmider, F.-X., Fossat, E., \& Mosser, B. 1991, A\&A, 248, 281

Stevenson, D. J., \& Salpeter, E. E. 1976, in Jupiter, ed. T. Gehrels (University of Arizona Press)

Walter, C. M., Marley, M. S., Hunten, D. M., et al. 1996, Icarus, 121,341

Zharkov, V. N., \& Trubitsyn, V. P. 1976, in Jupiter, ed. T. Gehrels (University of Arizona Press) 